\title{
Some 2-point sets
}

by

\author{
James H. Schmerl (Storrs, CT)
}

\begin{abstract}
Chad, Knight \& Suabedissen [Fund. Math. 203 (2009)] recently proved, assuming $\mathrm{CH}$, that there is a 2-point set included in the union of countably many concentric circles. This result is obtained here without any additional set-theoretic hypotheses.
\end{abstract}

A 2-point set is a subset of the plane $\mathbb{R}^{2}$ that meets every line at exactly 2 points. Mazurkiewicz [5] gave the first construction of a 2-point set. There have been other constructions of 2-point sets such as in [3] where it was shown that they exist in arbitrary vector spaces over arbitrary infinite fields. Recently, Chad, Knight \& Suabedissen [1] proved that the Continuum Hypothesis implies that there is a 2-point set included in the union of countably many concentric circles. Their conclusion is even stronger, being precisely the statement of the Theorem below. Subsequent to [1], Miller [6] constructed models of ZFC in which the continuum is arbitrarily large and there is a 2-point set that is included in the union of $\omega_{1}$ circles.

If $0<r \in \mathbb{R}$, then $C_{r}=\left\{x \in \mathbb{R}^{2}:\|x\|=r\right\}$ is the circle of radius $r$ centered at the origin. We prove the following theorem without any additional set-theoretic hypotheses.

THEOREM. Let $r_{0}, r_{1}, r_{2}, \ldots$ be a strictly increasing, unbounded sequence of positive real numbers. There is a 2-point set $M \subseteq \bigcup\left\{C_{r_{i}}: i<\omega\right\}$.

Proof. By replacing the sequence $r_{0}, r_{1}, r_{2}, \ldots$ with one of its subsequences, we can assume that one of the following holds:

(A) $\left\{r_{i}: i<\omega\right\}$ is algebraically independent.

(B) Whenever $i, j<\omega$, then $r_{i}$ is algebraic over $r_{j}$.

In the first part of this proof, Part I, we will give a proof of the Theorem assuming (A). In Part II, we modify Part I into a proof of the Theorem assuming (B).

2010 Mathematics Subject Classification: Primary 03E05; Secondary 03C64.

Key words and phrases: 2-point set. 
PART I: Assume (A). Let $\mathcal{S}=\left\{r_{i}: i<\omega\right\}$. Extend $\mathcal{S}$ to a transcendence basis $\mathcal{T} \supseteq \mathcal{S}$ for $\mathbb{R}$ over $\mathbb{Q}$. If $X \subseteq \mathbb{R}, n<\omega$ and $D \subseteq \mathbb{R}^{n}$, then we say that $D$ is $X$-definable if it is definable in the ordered field $(\mathbb{R},+, \cdot, 0,1, \leq)$ by a first-order formula that involves parameters only from $X$. By the TarskiSeidenberg Theorem (see [2]) on the elimination of quantifiers, if $a \in \mathbb{R}$, then $\{a\}$ is $X$-definable iff $a$ is algebraic over $X$. If $D \subseteq \mathbb{R}^{n}$ is $\mathbb{R}$-definable, then $\operatorname{supp}(D)$ (the support of $D$ ) is the unique smallest subset $X \subseteq \mathcal{T}$ such that $D$ is $X$-definable.

Let $\mathcal{L}$ be the set of all lines $\ell \subseteq \mathbb{R}^{2}$. For each $i<\omega$, define $L_{i} \subseteq \mathcal{L}$ so that $\ell \in L_{i}$ iff $i$ is the least such that:

(1) $\left|\ell \cap C_{r_{i}}\right|=2$,

(2) $\operatorname{supp}(\ell) \cap \mathcal{S} \subseteq\left\{r_{0}, r_{1}, \ldots, r_{i-1}\right\}$.

Clearly, each line is in exactly one $L_{i}$. Recursively define $M_{<i}$ and $M_{i}$ so that $M_{<i}=M_{0} \cup M_{1} \cup \cdots \cup M_{i-1}$ and $M_{i}$ is the set of those points $x \in \mathbb{R}^{2}$ such that there is a line $\ell \in L_{i}$ such that either:

(3) $\ell \cap M_{<i}=\emptyset$ and $x \in \ell \cap C_{r_{i}}$; or

(4) $\ell \cap M_{<i}$ is a singleton and $x$ is the first point of $\ell \cap C_{r_{i}}$ in the lexicographic ordering of $\mathbb{R}^{2}$.

Clearly, $M_{i} \subseteq C_{r_{i}}$ for each $i<\omega$. Let $M=\bigcup\left\{M_{i}: i<\omega\right\}$. Obviously, $M \subseteq \bigcup\left\{C_{r_{i}}: i<\omega\right\}$. We will show that $M$ is a 2-point set.

Consider an arbitrary line $\ell \in \mathcal{L}$, and let $i<\omega$ be such that $\ell \in L_{i}$. We want to show that $|\ell \cap M|=2$. Clearly, (1), (3) and (4) imply that $\left|\ell \cap M_{<i}\right|+\left|\ell \cap M_{i}\right| \geq 2$, so $|\ell \cap M| \geq 2$.

It remains to show that $|\ell \cap M| \leq 2$. Suppose the contrary, and let $j<\omega$ be the least such that $\left|\ell \cap M_{\leq j}\right| \geq 3$. [Notation: $M_{\leq j}=M_{<j} \cup M_{j}$.]

Suppose $j=i$. It follows from (3) and (4) that in order for $\left|\ell \cap M_{\leq i}\right| \geq 3$, it must be that there are $w \in C_{r_{i}}$ and $\ell^{\prime} \in L_{i}$ such that $\ell \cap \ell^{\prime}=\{w\}$. But then $r_{i}$ is $\left(\operatorname{supp}(\ell) \cup \operatorname{supp}\left(\ell^{\prime}\right)\right)$-definable, so $r_{i} \in \operatorname{supp}(\ell) \cup \operatorname{supp}\left(\ell^{\prime}\right)$, contradicting (2).

Thus, $j \neq i$ and, as in the previous paragraph, there are $w \in C_{r_{j}}$ and $\ell^{\prime} \in L_{j}$ such that $\ell \cap \ell^{\prime}=\{w\}$. Therefore, $r_{j} \in \operatorname{supp}(\ell) \cup \operatorname{supp}\left(\ell^{\prime}\right)$. Clearly, (2) implies that $j<i$ and $r_{j} \notin \operatorname{supp}\left(\ell^{\prime}\right)$, so $r_{j} \in \operatorname{supp}(\ell)$. Since $\left|\ell \cap M_{\leq j}\right| \geq 3$ and $\left|\ell \cap M_{j}\right| \leq 2$, it must be that $\left|\ell \cap M_{<j}\right| \in\{1,2\}$.

First, suppose that $\left|\ell \cap M_{<j}\right|=2$, and let $y, z \in \ell \cap M_{<j}$ be distinct. Then, since $r_{j} \in \operatorname{supp}(\ell)$, it follows that $r_{j} \in \operatorname{supp}(\{y, z\})$, so assume $r_{j} \in \operatorname{supp}(y)$. Let $k<j$ be such that $y \in M_{k}$. Then there is $\ell^{\prime \prime} \in L_{k}$ such that $y \in \ell^{\prime \prime} \cap C_{r_{k}}$, so $r_{j} \in \operatorname{supp}\left(\ell^{\prime \prime}\right) \cup\left\{r_{k}\right\}$, contradicting (2).

Second, suppose that $\left|\ell \cap M_{<j}\right|=1$, and let $\ell \cap M_{<j}=\{x\}$. Then there is $k<j$ such that $x \in M_{k}$. Let $\ell \cap M_{j}=\{y, z\}$ and let $\ell^{\prime}, \ell^{\prime \prime} \in L_{j}$ be such that $y \in \ell^{\prime} \cap C_{r_{j}}$ and $z \in \ell^{\prime \prime} \cap C_{r_{j}}$. It then follows from the following lemma 
that $r_{j}$ is $\left(\operatorname{supp}\left(\ell^{\prime}\right) \cup \operatorname{supp}\left(\ell^{\prime \prime}\right) \cup \operatorname{supp}(x)\right)$-definable, contradicting (2) and thereby completing the proof assuming (A).

Lemma 1. Suppose that $\ell^{\prime}, \ell^{\prime \prime} \in \mathcal{L}$ are distinct and $x \in \mathbb{R}^{2} \backslash\left(\ell^{\prime} \cup \ell^{\prime \prime}\right)$. Then there are at most finitely many $r>0$ such that there are $y \in \ell^{\prime} \cap C_{r}$ and $z \in \ell^{\prime \prime} \cap C_{r}$ with $x, y, z$ being collinear.

Lemma 1 is Lemma 4.1 of [1]. As stated in [1], it says that there are at most 23 possible $r$; this does not seem to be the optimal number.

Before starting Part II of this proof, we prove a simple lemma.

LEMma 2. Let $\varphi\left(y_{0}, y_{1}, \ldots, y_{m-1}, u_{0}, u_{1}, \ldots, u_{n-1}, x\right)$ be a formula in the language of ordered fields, and let $a_{0}, a_{1}, \ldots, a_{m-1} \in \mathbb{R}$. Then there are only finitely many $b \in \mathbb{R}$ that are algebraic over $\left\{a_{0}, a_{1}, \ldots, a_{m-1}\right\}$ for which there are $t_{0}, t_{1}, \ldots, t_{n-1} \in \mathbb{R}$ that are algebraically independent over $\left\{a_{0}, a_{1}, \ldots, a_{m-1}\right\}$ such that $\varphi(\bar{a}, \bar{t}, x)$ defines $b$ in $\mathbb{R}$.

Proof. This lemma is a consequence of the o-minimality of $\mathbb{R}$ considered as an ordered field. (See [2, Chapter 2].) Let $f: D \rightarrow \mathbb{R}$, where $D \subseteq \mathbb{R}^{n}$, be the $\left\{a_{0}, a_{1}, \ldots, a_{n-1}\right\}$-definable function such that whenever $d_{0}, d_{1}, \ldots, d_{m-1}, b \in \mathbb{R}$ are such that $\varphi(\bar{a}, \bar{d}, x)$ defines $b$, then $\bar{d} \in D$ and $f(\bar{d})=b$. If $t_{0}, t_{1}, \ldots, t_{n-1} \in \mathbb{R}$ are algebraically independent over $\left\{a_{0}, a_{1}\right.$, $\left.\ldots, a_{m-1}\right\}, \bar{t} \in D$ and $f(\bar{t})=b$ is algebraic over $\left\{a_{0}, a_{1}, \ldots, a_{m-1}\right\}$, then $f$ is constantly $b$ on some neighborhood $U$ of $\bar{t}$. If $b_{0}, b_{1}, b_{2}, \ldots$ are infinitely many distinct such possibilities for $b$, then there are corresponding neighborhoods $U_{0}, U_{1}, U_{2}, \ldots \subseteq D$ such that $f$ is constantly $b_{i}$ on $U_{i}$. But this is impossible by o-minimality.

PART II: Assume (B). If $r_{0}$ is algebraic, let $\mathcal{S}=\emptyset$, and if $r_{0}$ is transcendental, let $\mathcal{S}=\left\{r_{0}\right\}$. As in Part I, extend $\mathcal{S}$ to a transcendence basis $\mathcal{T}$. With Lemma 2 in mind, we make the following ad hoc definition. If $A \subseteq\left\{r_{0}, r_{1}, r_{2}, \ldots\right\}$ and $i<\omega$, then $\operatorname{Def}(A, i)$ is the set of all $\mathbb{R}$-definable $D \subseteq \mathbb{R}^{n}$, for some $n<\omega$, such that $D$ is $(\mathcal{T} \cup A)$-definable by a formula having length at most $i$.

We now recursively get a subsequence $r_{k_{0}}, r_{k_{1}}, r_{k_{2}}, \ldots$ that is sufficiently fast growing. To be definitive, let $k_{0}=0$, and then let $k_{i+1}$ be the least $k>k_{i}$ such that the following hold, where $A=\left\{r_{j}: j \leq k_{i}\right\}$ :

(5) If $\ell, \ell^{\prime} \in \mathcal{L} \cap \operatorname{Def}(A, i+1), \ell \cap \ell^{\prime}=\{w\}, r=\|w\|$ and $r$ is algebraic over $\left\{r_{0}\right\}$, then $r_{k}>r$.

(6) [cf. Lemma 1] If $\ell^{\prime}, \ell_{1}, \ell_{2} \in \mathcal{L} \cap \operatorname{Def}(A, i+1), w \in \ell^{\prime},\|w\| \in A$, $w \notin \ell_{1} \cup \ell_{2}, y \in \ell_{1}, z \in \ell_{2}, w, y, z$ are collinear, $\|y\|=\|z\|=r$ and $r$ is algebraic over $r_{0}$, then $r_{k}>r$.

Lemma 2 guarantees that $k_{i+1}$ is well defined. For notational convenience and without loss of generality, we will assume that $r_{k_{i}}=r_{i}$ for all $i<\omega$. 
For each $i<\omega$, define $L_{i} \subseteq \mathcal{L}$ so that $\ell \in L_{i}$ iff $i$ is the least such that (1) and the following hold:

(7) $\ell \in \operatorname{Def}\left(\left\{r_{0}, r_{1}, \ldots, r_{i-1}\right\}, i\right)$.

Clearly, each line is in exactly one $L_{i}$. Notice that the set $\mathcal{L} \cap \operatorname{Def}(A, i+1)$ occurring in (5) and (6) is $L_{0} \cup L_{1} \cup \cdots \cup L_{i+1}$. Define $M_{<i}$ and $M_{i}$ just as in (3) and (4) in Part I, and then define $M$ the same way.

Again, $M_{i} \subseteq C_{r_{i}}$, so $M \subseteq \bigcup\left\{C_{r_{i}}: i<\omega\right\}$. We will show that $M$ is a 2-point set by an argument that parallels the one in Part I.

Consider some $i<\omega$ and an arbitrary $\ell \in L_{i}$. As in Part I, $|\ell \cap M| \geq 2$, so it remains to show that $|\ell \cap M| \leq 2$. Suppose the contrary, and let $j<\omega$ be the least such that $\left|\ell \cap M_{\leq j}\right| \geq 3$.

Suppose $j=i$. It follows from (3) and (4) that in order for $\left|\ell \cap M_{\leq i}\right| \geq 3$, it must be that there are $w \in C_{r_{i}}$ and $\ell^{\prime} \in L_{i}$ such that $\ell \cap \ell^{\prime}=\{w\}$. But this contradicts (5).

Thus, $j \neq i$ and there are $w \in C_{r_{j}}$ and $\ell^{\prime} \in L_{j}$ such that $\ell \cap \ell^{\prime}=\{w\}$. So again by (5), it cannot be that $j>i$. Thus $j<i, w \in C_{r_{j}}$ and $\left|\ell \cap M_{j}\right| \leq 2$, so it must be that $\left|\ell \cap M_{<j}\right| \in\{1,2\}$.

First, suppose that $\left|\ell \cap M_{<j}\right|=2$, and let $\{y, z\}=\ell \cap M_{<j}$. Let $k_{1}, k_{2}<j$, $\ell_{1} \in L_{k_{1}}$ and $\ell_{2} \in L_{k_{2}}$ be such that $y \in \ell_{1} \cap C_{r_{k_{1}}}$ and $z \in \ell_{2} \cap C_{r_{k_{2}}}$. Clearly, this contradicts (6).

Second, suppose that $\left|\ell \cap M_{<j}\right|=1$, and let $\ell \cap M_{<j}=\{x\}$. Then, there is $k<j$ such that $x \in M_{k}$. Let $\ell \cap M_{j}=\{y, z\}$ and let $\ell_{1}, \ell_{2} \in L_{j}$ be such that $\{y\}=\ell \cap \ell_{1}$ and $\{z\}=\ell \cap \ell_{2}$. Clearly, this contradicts (6), completing Part II and the proof of the Theorem.

A long-standing open problem (see [4]) is whether there is a Borel 2-point set. Very closely related to this is the question: Can the existence of a 2-point set be proved in ZF (that is, ZFC with the Axiom of Choice deleted). In the absence of a positive answer to this last question, one can ask for weak consequences of $\mathrm{AC}$ that imply the existence of a 2-point set. The following is an example of such a consequence, although I am unable to say what its strength is relative to other consequences:

(*) There is a real-closed subfield $\mathbb{F} \subseteq \mathbb{R}$ such that the transcendence degree of $\mathbb{R}$ over $\mathbb{F}$ is $\aleph_{0}$.

The following is a consequence of Part I of the proof of the Theorem.

Corollary. (ZF) If (*), then there is a 2-point set.

Proof. Let $\mathbb{F}$ be as in (*). Let $\mathcal{S}=\left\{r_{0}, r_{1}, r_{2}, \ldots\right\}$ be a transcendence basis for $\mathbb{R}$ over $\mathbb{F}$ such that $r_{0}, r_{1}, r_{2}, \ldots$ is a strictly increasing, unbounded sequence of positive real numbers. In Part I of the proof of the Theorem, we would extend $\mathcal{S}$ to a transcendence basis $\mathcal{T} \supseteq \mathcal{S}$ for $\mathbb{R}$ over $\mathbb{Q}$. We see 
that the construction in the proof is independent of the actual choice of $\mathcal{T}$. In the absence of $A C$, it may be impossible to get any such $\mathcal{T}$; however, if we modify the definition of support so that $\operatorname{supp}(D)$ is $X \cup \mathbb{F}$, where $X$ is the smallest subset $X \subseteq \mathcal{S}$ such that $D$ is $(X \cup \mathbb{F})$-definable, then the proof still works.

\section{References}

[1] B. Chad, R. Knight, and R. Suabedissen, Set-theoretic constructions of two-point sets, Fund. Math. 203 (2009), 179-189.

[2] L. van den Dries, Tame Topology and o-Minimal Structures, London Math. Soc. Lecture Note Ser. 248, Cambridge Univ. Press, 1998.

[3] P. Komjáth and J. H. Schmerl, The two points theorem of Mazurkiewicz, J. Combin. Theory (A) 99 (2002), 371-376.

[4] R. D. Mauldin, Problems in topology arising from analysis, in: Open Problems in Topology, North-Holland, Amsterdam, 1990, 617-629.

[5] S. Mazurkiewicz, Sur un ensemble plan, C. R. Acad. Sci. Lettres Varsovie 7 (1914), $382-384$.

[6] A. W. Miller, The axiom of choice and two-point sets in the plane, http://www.math. wisc.edu/ $\sim$ miller/res

James H. Schmerl

Department of Mathematics

University of Connecticut

Storrs, CT 06269-9003, U.S.A.

E-mail: schmerl@math.uconn.edu 